12

\title{
Повышение эффективности систем дистанционного радиационного контроля на базе линейного ускорителя электронов
}

\author{
(C) Б.Ю. Богданович, Г.О. Буянов, А.В. Нестерович \\ Национальный исследовательский ядерный университет „МИФИ“, \\ 115409 Москва, Россия \\ e-mail: frontlines.07@gmail.com
}

Поступило в Редакцию 26 октября 2020 г.

В окончательной редакции 9 фревраля 2021 г.

Принято к публикации 11 февраля 2021 г.

Рассмотрена система предварительного „охлаждения“ пучка электронов и уменьшения угловой расходимости потока квантов за счет многократного прохождения пучка электронов в камере, помещенной в поперечное магнитное поле. Охлаждение электронного пучка обеспечивается специальной конфигурацией магнитного поля. В предложенной схеме фазовый портрет претерпевает простейшие изменения, а именно, в фольге скачкообразно возрастает, как бы „вытягиваясь“ в вертикальном направлении, но затем как бы „вращается“ вокруг начала координат, при этом достигая горизонтального положения после „фокусировки“. Таким образом, поперечные размеры пучка возрастают, а угловой разброс по крайней мере не увеличивается, как без охлаждения.

Ключевые слова: интроскопия, томография, тормозное излучение, выходное устройство, электронный пучок.

DOI: $10.21883 /$ JTF.2021.07.50959.298-20

\section{Введение}

Актуальность работ по системам инспекционного назначения подтверждает нескончаемый поток наркотиков и взрывчатых веществ, значительная часть которых пересекает границу сухопутными и морскими путями (не говоря о воздушном) [1-5].

Ускорительные комплексы мобильного базирования требуют совершенствования в части создания эффективных выходных устройств. Нетрудно видеть, что потеря более 90\% фотонного пучка в коллиматорах на выходе ускорителя электронов делает время экспозиции на 2 порядка больше, чем без „обрезания“ пучка, что возможно в рассматриваемой схеме. Данное техническое решение позволяет во столько же раз (до 2 порядков) сократить время облучения, в том числе персонала, обслуживающего установку, и близлежащих объектов. Набор параллельных пучков излучения, имеющих в совокупности ограниченный размер, может менять направление в достаточно широком угловом диапазоне без громоздких и тяжеловесных коллиматоров.

Системы, обеспечивающие формирование пучка тормозного излучения малой расходимости за счет многократного прохождения через тонкую фольгу с одновременным „охлаждением“ электронного пучка в магнитном поле специальной конфигурации, предложены и испытаны ранее $[1,6]$. При этом максимальная энергия пучка достигала $4.5 \mathrm{MeV}$. Дозиметрические измерения показали, что метод многократного прохождения электронами тонкой конверсионной мишени увеличивает выход тормозного излучения (ТИ) примерно в 2 раза по сравнению с толстой мишенью оптимальной толщи- ны [6]. Задача повышения эффективности таких систем особенно остро встает в связи с увеличением требований к энергии пучка, а именно, с необходимостью определения состава инспектируемого груза на предмет наличия взрывчатых веществ, т.е. с возрастанием энергии электронного пучка до нескольких десятков $\mathrm{MeV}$ [2]. Первичная коллимация пучка такой энергии методом диафрагмирования не дает желаемых результатов. Кроме того, необходимо увеличение глубины зондирования, что также требует возрастания энергии выше известного предела (около $10 \mathrm{MeV}$ ) [3]. Практическое использование гамма-излучения для получения прямых данных о содержании скрытых взрывчатых веществ стало возможным благодаря развитию ускорительной техники, созданию компактных ускорителей электронов с энергией до $100 \mathrm{MeV}$ и генерации с их помощью мощных пучков гамма-излучения высоких энергий, при которых начинают ярко проявляться специфические для химических элементов взрывчатых веществ фотоядерные эффекты.

Принцип действия данной системы апробирован экспериментально и получил развитие в работах по формированию потоков тормозного излучения в системах широкоапертурного типа [4-6].

В работе [4] изложена суть метода „охлаждения“ пучка электронов и получения потока ТИ с уменьшенной угловой расходимостью в одном из вариантов в технической реализации, а именно, в системе, образованной последовательностью тонких конвертеров, установленных ступенчатым образом (так называемая „лесенка“), разделенных парами магнитных дефлекторов, обеспечивающих параллельный перенос пучка с одновременной фокусировкой. В данном случае наибо- 
лее близким аналогом (прототипом) является система, описанная в работе [6]. Она представляет собой магнитную систему, обеспечивающую многократное вращение пучка электронов при прохождении через конвертер, установленный параллельно оси ускорителя, так что поток $\gamma$-квантов направлен перпендикулярно этой оси, что не всегда удобно с точки зрения решения задач дистанционного контроля. Фокусировка пучка обеспечивается за счет градиента магнитного поля, так что расходимость пучка перед входом в конвертер минимизируется. На ускорителе с энергией электронов (3-6) MeV достигнуто двукратное увеличение выхода тормозного излучения по сравнению с максимумом для мишени оптимальной толщины. В настоящей работе рассмотрена система, позволяющая получить пучок ТИ в направлении оси ускорителя, и существенно (в несколько раз) увеличить выход ТИ с малой расходимостью за счет оптимального выбора параметров магнитного поля и конвертора.

\section{1. Принципиальная схема выходного устройства}

На рис. 1 показана схема выходного устройства, формирующего потоки тормозного излучения $\left(\tilde{\gamma}_{1}, \tilde{\gamma}_{2}, \ldots, \gamma_{i}\right)$, параллельные и остронаправленные. Мишени $M_{i}(i=1, \ldots, J)$ по толщине существенно меньше (приблизительно в 30-40 раз), чем „оптимальная“ толщина мишени из того же материала (медь, алюминий

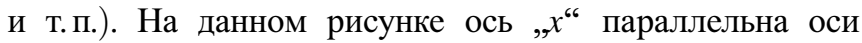
пучка электронов до попадания в выходное устройство, а центр вакуумной камеры совмещен с осью вращения электронов в магнитном поле с радиусом $R_{1}$ (можно

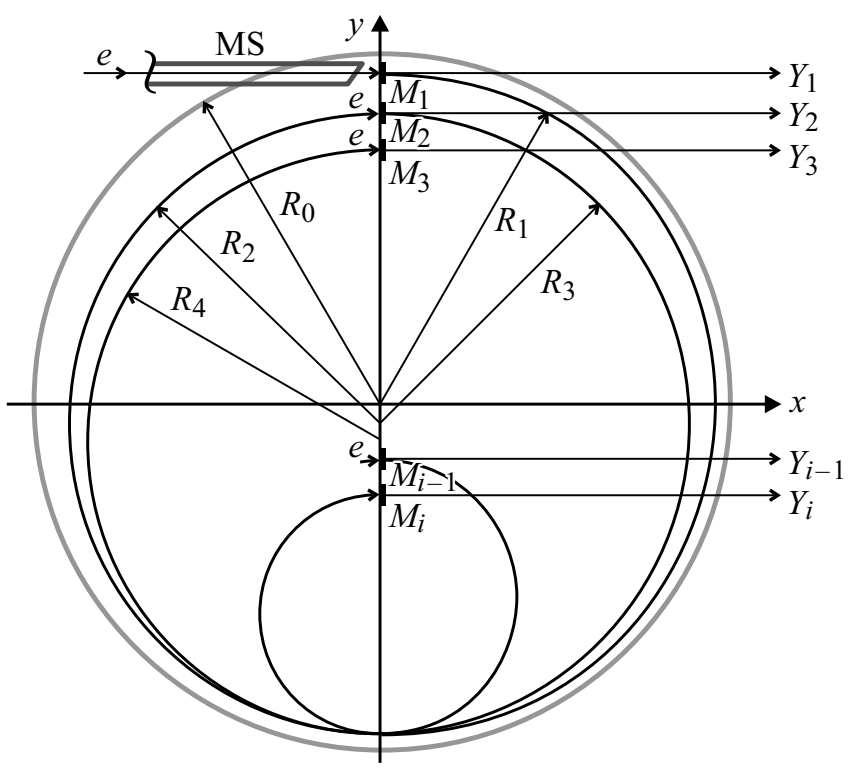

Рис. 1. Принципиальная схема выходного устройства (пояснения в тексте).

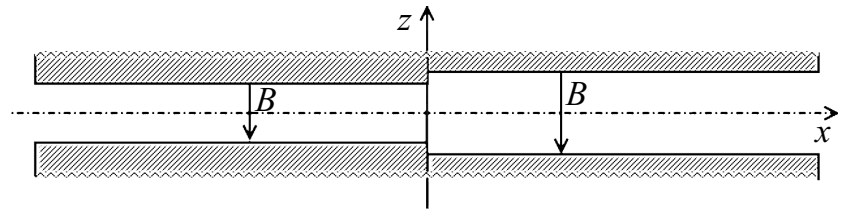

Рис. 2. Вид межполюсного зазора магнитов.

считать ее осью системы, перпендикулярной плоскости вращения).

Электроны, выходя из линейного ускорителя электронов с энергией в несколько десятков $\mathrm{MeV}$, попадают в поперечные однородные (в первом приближении) магнитные поля с индукцией порядка 1T.

Проходя через мишени $M_{i}$, электроны теряют часть своей энергии, радиус их вращения в правой половине магнитного поля (если смотреть на чертеж) незначительно больше, чем в левой за счет несколько меньшей величины индукции.

Это происходит благодаря тому, что межполюсной зазор справа больше, чем в левой половине (рис. 2). Таким образом, уже первая орбита электронов не задевает трубку из магнитомягкого материала, по которой проходит пучок в магнитное поле („магнитный экран“ MS). Во второй мишени $M_{2}$ пучок также теряет энергию на тормозное излучение и ионизацию и двигается по траектории, радиус которой определяется величиной потери энергии на фольге $M_{2}$ и меньшим магнитным полем, чем до фольги.

В зависимости от соотношения этих величин траектория электрона может проходить нижнюю точку „a“, через которую электрон проходил на первом обороте, а может проходить выше или ниже. Как показывает проведенный анализ, оптимальным является случай, когда все траектории проходят через точку „, $а$ “ или возле нее. Нетрудно видеть, что, например, в противоположенном случае или система оказывается неоправданно „вытянутой“ вдоль оси „Y“, или траектории „наползают“ друг на друга и на соседние мишени.

Условие прохождения всех траекторий через точку „ $a$ “ соответствует равенству изменения диаметра вращения от изменения энергии в фольге $\delta D_{F i}$ и от изменения магнитного поля $\delta D_{B i}$, т. е.

$$
\delta D_{F i}=\delta D_{B i}=\delta D_{i} .
$$

Для релятивистского пучка

$$
\frac{\delta D_{i}}{D_{i}}=\frac{\Delta B}{B}=v=\mathrm{const}
$$

где $\Delta B$ - разница в значении индукции в левой и правой половине зоны локализации магнитного поля, образуемая разницей величины зазора.

Значения $D_{i}$ связаны соотношением

$$
D_{i+1}=D_{i}-\delta D_{i}=D_{i}\left(1-\frac{\delta D_{i}}{D_{i}}\right)=D_{i}(1-v) .
$$


В свою очередь, значения $D_{i}$ и $\gamma_{i}$ связаны посредством уравнения

$$
\frac{\gamma_{i+1}}{\gamma_{i}}=\frac{D_{i+1}}{D_{i}}=\frac{D_{i}-\delta D_{i}}{D_{i}}=1-\frac{\delta D_{i}}{D_{i}}=1-v,
$$

$\Delta \gamma_{i}=\gamma_{i+1}-\gamma_{i}=\gamma_{i}\left(\frac{\gamma_{i+1}}{\gamma_{i}}\right)-1=\gamma_{i}(1-v-1)=-\gamma_{i} v$,

где $\gamma$ - относительная энергия электрона, или Лоренцфактор.

Для соотношения $\gamma_{I}$ и $v$ имеем

$$
\frac{\gamma_{I}}{\gamma_{1}}=\frac{\gamma_{2}}{\gamma_{1}} \frac{\gamma_{3}}{\gamma_{2}}, \ldots, \frac{\gamma_{I}}{\gamma_{I-1}}=(1-v)^{I} \approx 1-v I
$$

$(v \ll 1)$, или в окончательном виде

$$
I=\left|\frac{\lg \varkappa}{\lg (1-v)}\right|, \quad \text { где } \quad \varkappa=\frac{\gamma_{i}}{\gamma_{0}},
$$

где знак $|\ldots|$ означает округление до целочисленного значения. Это соотношение удобно использовать при предварительном выборе параметров системы, исходя из общих соображений.

В частности, при $\varkappa=\frac{\gamma I}{\gamma_{1}}=\frac{1}{4}\left(\frac{R_{I}}{R_{1}}=\frac{1}{4}\right), \quad$ и при $v=3 \cdot 10^{-2}$ имеем $\dot{I}=45(45$ мишеней $M)$, при $\varkappa=\frac{1}{3}$ получаем $\dot{I}=36$.

Рассмотрим, что происходит с пучком электронов при прохождении в данной системе.

\section{2. Прохождение электронного пучка}

Электроны, получившие импульс в направлении оси , ,“, беспрепятственно полетят в направлении крышек вакуумной камеры, где и будут потеряны. Не лучше ситуация с электронами, получившими радиальный импульс (рис. 3, 4, электроны $e_{1}-e_{4}$ ).

Для того чтобы направить электроны перпендикулярно поверхности фольги $M$, необходимо изменить их

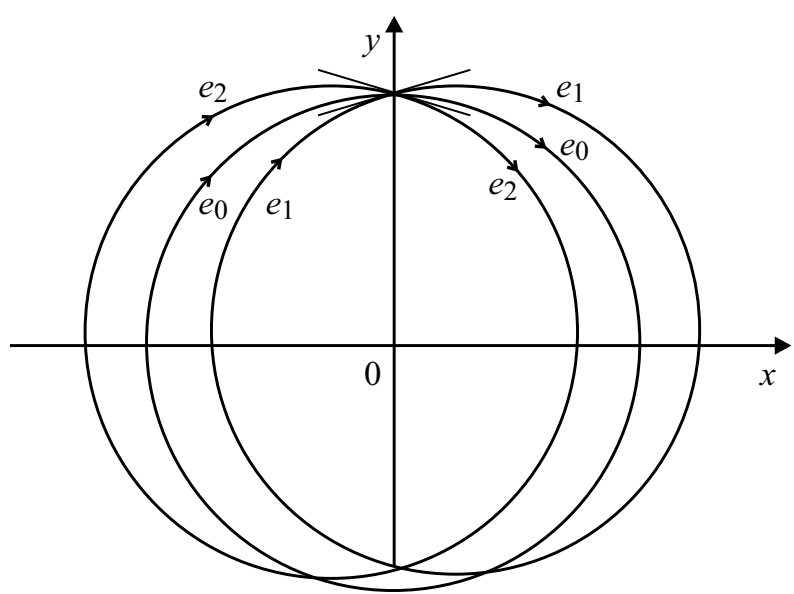

Рис. 3. Принципиальная схема траекторий электронов в медианной плоскости.

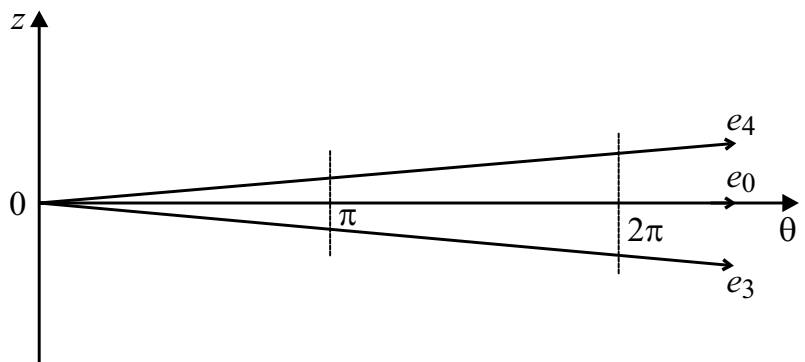

Рис. 4. Принципиальная схема траекторий электронов в вертикальной плоскости (перпендикулярной плоскости вращения (без фокусировки и охлаждения)).

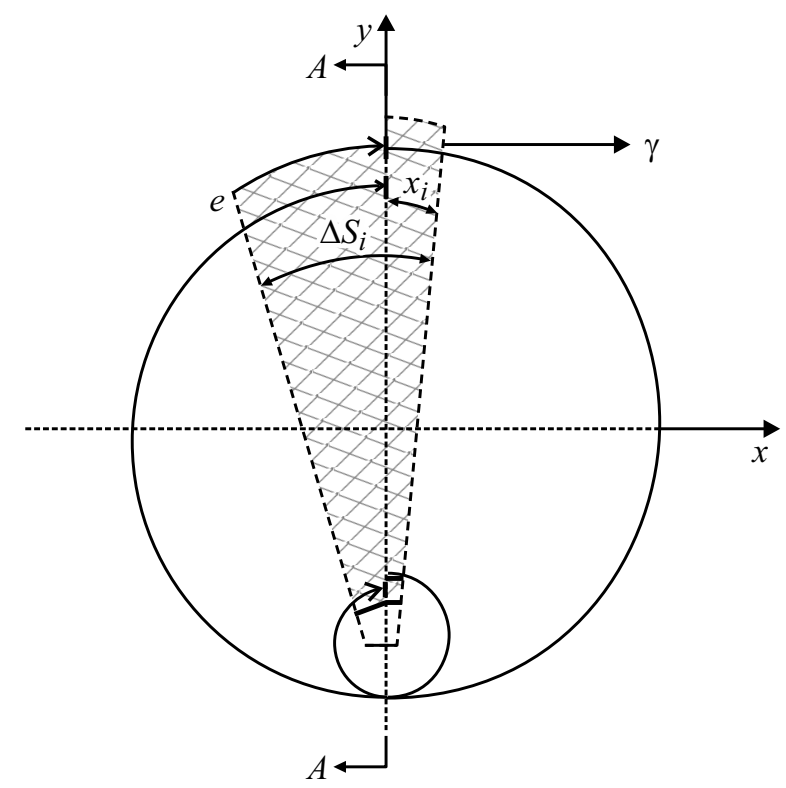

Рис. 5. Зона локализации градиентного поля (пояснения в тексте).

траектории в соответствии с условиями фокусировки. Анализируя движение электронов, замечаем, что если в направлении оси „, $z^{6}$ угловое расхождение сохраняется первоначальным, а пучок является расходящимся, как и при вылете из предыдущей фольги, то в радиальном направлении, в частности, вдоль оси „у“, пучок является сходящимся и влетает в последующую фольгу при том же угловом разбросе (на рис. 3 для простоты изложения показан случай без перепада индукции между половинками магнитной зоны).

Очевидно, что для компенсации углового разброса, т. е. для формирования пучка с параллельными траекториями, необходим квадрупольный магнит. Роль такого магнита может выполнить градиентное магнитное поле, являющееся, по существу, суперпозицией однородного и квадрупольного полей [7].

На рис. 5 изображена зона локализации такого поля (заштриховано), расположенная перед влетом электронов в фольгу (общий случай). На рис. 6 показано сечение 


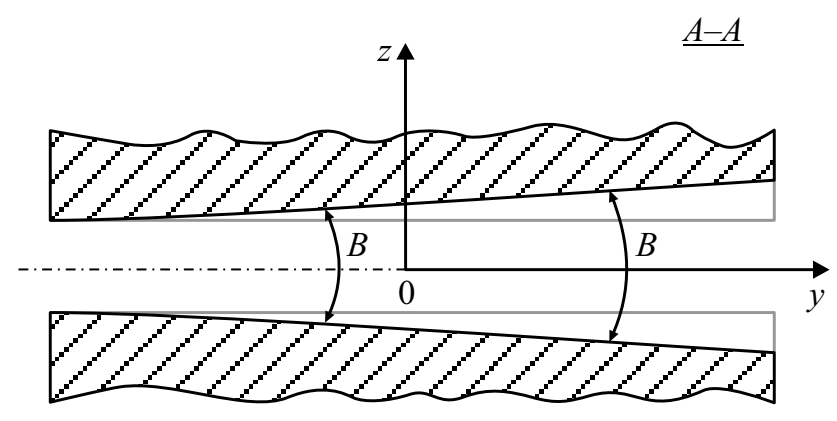

Рис. 6. Сечение магнитных полюсов в области градиента магнитного поля.

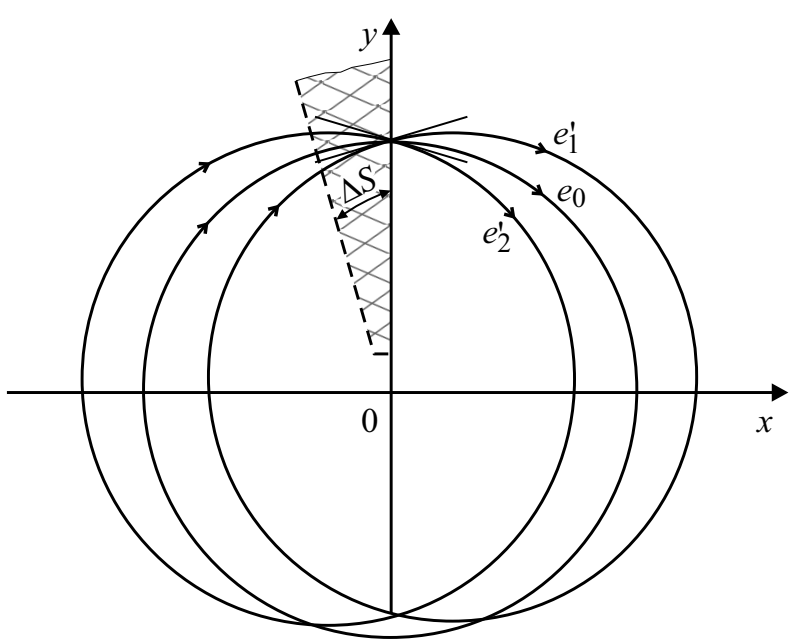

Рис. 7. Траектории электронов в области градиента поля $(x, y-$ координаты в медианной плоскости).

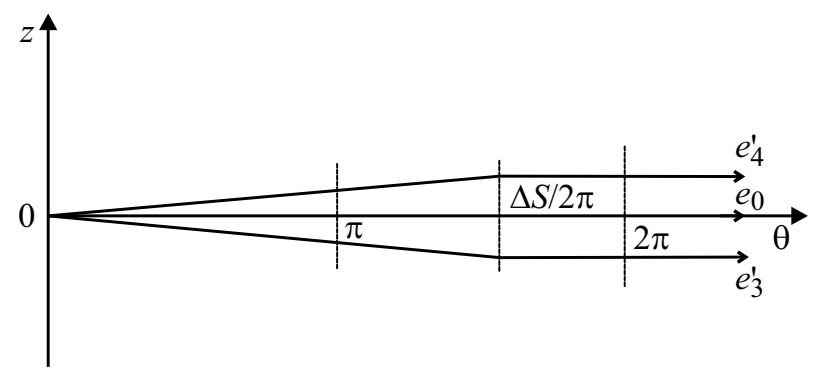

Рис. 8. Траектория электронов поля в области градиента $(z, \theta-$ координаты в вертикальной плоскости).

магнитных полюсов в этой зоне, а на рис. 7 и 8 характерные траектории электронов при наличии такой зоны с градиентом поля (упрощенный варинат).

Оценим величину градиента магнитного поля для диапазона энергий несколько десятков $\mathrm{MeV}$.

В общем случае частица, получившая импульс $P_{\perp}$ (независимо по оси „у“ или , $x^{“}$ ), влетает в фольгу, совершив 1 оборот, под углом $\alpha$ на расстоянии $r^{*}$

$$
\alpha=\frac{P_{\perp}}{P}=\frac{\Delta R}{2 \pi R_{0}} .
$$

Для компенсации углового отклонения необходим такой же импульс

$$
P_{\perp}=F_{\perp} \Delta t=e \Delta B \Delta S
$$

Отсюда получаем

$$
|n| \approx \frac{R_{0}}{B_{0}} \frac{\Delta B}{\Delta R}=\frac{\gamma c}{B_{0} \eta_{e o} 2 \pi} \frac{1}{\Delta S}
$$

в частности, для $W_{e}=100 \mathrm{MeV}(\gamma \approx 200), B_{0}=0.8 \mathrm{~T}$, $\Delta S=\Delta S_{1}=0.3 \mathrm{~m}$, имеем $n \approx 0.217$.

Для практической реализации можно рассмотреть случай, когда скос магнитного полюсного наконечника осуществляется от зазора порядка (35-36) $\mathrm{mm}$ на внешней поверхности магнита $\left(R_{o}=30 \mathrm{~cm}\right)$ до зазора $\approx(25-26)$ mm при $R_{25}=7.5 \mathrm{~cm}$.

Для расчета величин $\Delta S_{i}$ уравнение (10) перепишем в виде

$$
\Delta S_{i}=\mu \gamma_{i}
$$

где

$$
\mu=\frac{c}{2 \pi \eta_{e o} B_{0} n} .
$$

Для приведенного выше случая

$$
\mu=\frac{2.71 \cdot 10^{-4}}{n B_{0}}=1.56 \cdot 10^{-3} .
$$

Выражая $\Delta S_{i}$ в $[\mathrm{cm}]$, получим

$$
\Delta S_{i}=0.156 \gamma_{i}[\mathrm{~cm}]
$$

Для определения формы и размеров полюсных наконечников в зоне с неоднородным полем (с градиентом В) воспользуемся определением показателя спада магнитного поля:

$$
n=-\frac{r_{0}}{B_{0}} \frac{d B_{z}}{d r}
$$

где $B_{0}-$ среднее значение индукции в зоне локализации орбиты электрона с радиусом вращения $r_{0}$. В этой зоне (по размерам примерно равной расстоянию $z_{0}$ между полюсами магнита) можно считать

$$
\frac{B_{0}+\Delta B}{B_{0}}=\frac{z_{0}-\Delta z}{z_{0}}
$$

или

$$
\frac{\Delta B}{B_{0}} \approx-\frac{\Delta z_{0}}{z_{0}}
$$

Учитывая (11), получим

$$
\Delta z=(n) \frac{\Delta r}{R r_{0}} z_{0}
$$

Проводя вычисления для различных значений $r_{0}$ (в нашем случае $n=0.217, z_{0}$ изменяется от 7.5 до $30 \mathrm{~cm}$ ) и 


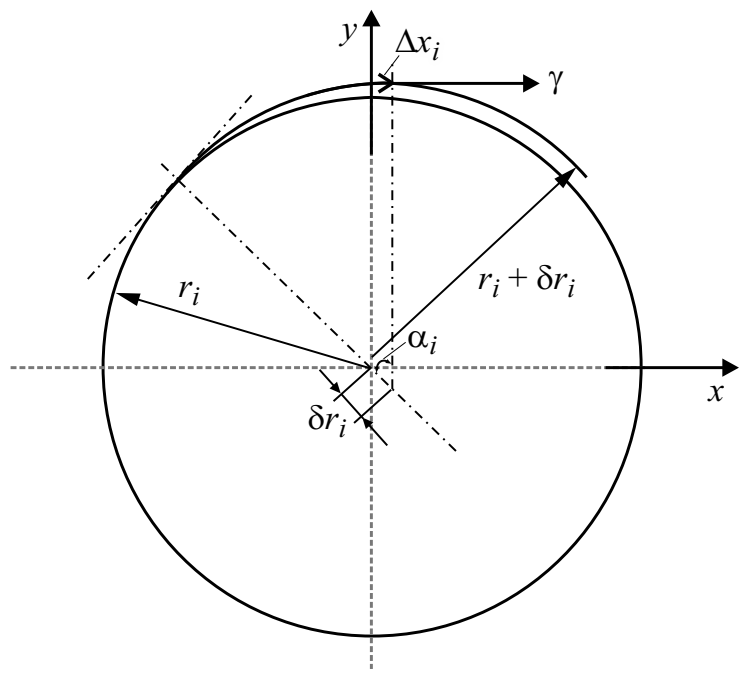

Pис. 9. Принципиальная схема прохождения электрона в области действия магнитного поля.

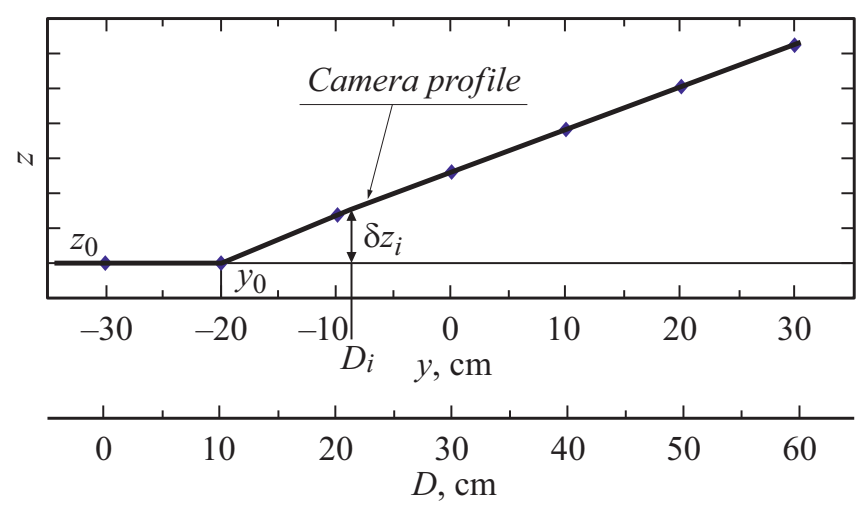

Рис. 10. Изменение профиля наконечника.

обобщая полученные результаты, получим приближенное соотношение для определения формы наконечника

$$
\begin{gathered}
\frac{\Delta z\left(y-y_{0}\right)}{\Delta y} \approx 2.7 \cdot 10^{-2}=\varkappa, \\
y_{0}=-20[\mathrm{~cm}], \\
z_{0}\left(y_{0}\right)=2.6[\mathrm{~cm}],
\end{gathered}
$$

$\Delta z_{0}(y=30 \mathrm{~cm})=2.7 \cdot 10^{-2} \cdot 50[\mathrm{~cm}]=1.35 \mathrm{~cm}$, $z_{0}(y=30 \mathrm{~cm})=2.6 \mathrm{~cm}+1.35 \mathrm{~cm}=3.95 \mathrm{~cm}(\approx 4 \mathrm{~cm})$.

Технически скос полюсного наконечника представляется возможным осуществить фрезерованием поверхности под постоянным углом с центром, совпадающим с центром наименьшей орбиты диаметром $15 \mathrm{~cm}$ по оси , $y^{\text {“ }}$, перепад высот на каждом $\leq 7 \mathrm{~mm}$.

Безусловно, данное обстоятельство влияет на искажение орбиты в целом на участке перед влетом в мишень, так что параллельность траекторий электронов не обеспечивается при прохождении всех мишеней на оси , $y^{\text {“ }}$ (рис. 9).
Для того чтобы вектор скорости каждого из электронов был параллелен оси ,x“ при прохождении фольги (или, по крайней мере, приближался к направлению оси), необходимо смещать точки влета вправо от оси , $x^{6}$ и рассматривать случай, когда медианная плоскость совпадает с плоскостью градиента. Смещение точки расположения мишени, в которой касательная к траектории параллельна оси , $x^{\text {с }}$, определяется величиной

$$
\Delta x_{i}=\delta r_{i} \sin \alpha
$$

где

$$
\alpha=\frac{\Delta S i}{\pi D_{i}}, \quad \delta r_{i}=r_{i} \frac{\delta B\left(r_{i}\right)}{B_{0}} .
$$

Учитывая, что

$$
\begin{gathered}
\frac{\delta B\left(r_{i}\right)}{B_{0}} \approx-\frac{\Delta z_{i}}{z_{0}}, \\
\Delta z_{i}=\varkappa\left(y_{i}-y_{0}\right),
\end{gathered}
$$

где $x=2.7 \cdot 10^{-2}$ (см. (14)), $y_{0}=-20[\mathrm{~cm}]$, получим $y_{i}=\left(D_{i}-30\right)[\mathrm{cm}]$.

Смысл преобразований поясняется на рис. 10, где показано изменение профиля наконечника для рассматриваемого варианта.

Значения $\Delta x_{i}$ для рассмотренного варианта сведены в график на рис. 11.

Точки на графике соединены отрезками прямых линий для наглядности. На том же рисунке показаны значения $\Delta S_{i}$ в зависимости от номера орбиты „іс

Для оценки величины коэффициента усиления $K_{\dot{I}}$ интенсивности потока фотонов на оси системы (рис. 1) в дальней зоне (без учета воздушной среды), т. е. при расстояниях $L \gg D_{0}$, может быть использовано выражение

$$
\begin{gathered}
K_{\dot{I}}=\frac{\sum_{i=1}^{i} \Delta \gamma_{i}\left[\lambda\left(\gamma_{i}\right)\right]\left(\gamma_{i}\right)^{2}}{\sum_{i=1}^{I} \Delta \gamma_{i}\left[\lambda\left(\lambda_{i}\right)\right]\left(\gamma_{i}^{*}\right)^{2}}=\frac{\sum_{i=1}^{i} \Delta \gamma_{i}\left[\lambda\left(\gamma_{i}\right)\right]\left(\gamma_{i}\right)^{2}}{\sum_{i=1}^{i} \frac{\Delta \gamma_{i}\left[\lambda\left(\lambda_{i}\right)\right]}{\sum_{i^{\prime}=1}^{i}\left(\frac{1}{\gamma_{i^{\prime}}}\right)^{2}}}, \\
\theta_{\gamma_{i}}^{*}=\sqrt{\sum_{i^{\prime}=1}^{i}\left(\frac{1}{\gamma_{j}^{i}}\right)^{2}}
\end{gathered}
$$

- угол расхождения потока квантов после $i$-го прохождения фольги без охлаждения, $\theta_{\gamma_{i}} \approx \frac{1}{\gamma_{i}}-$ угол расхождения потока квантов после $i$-го прохождения фольги с

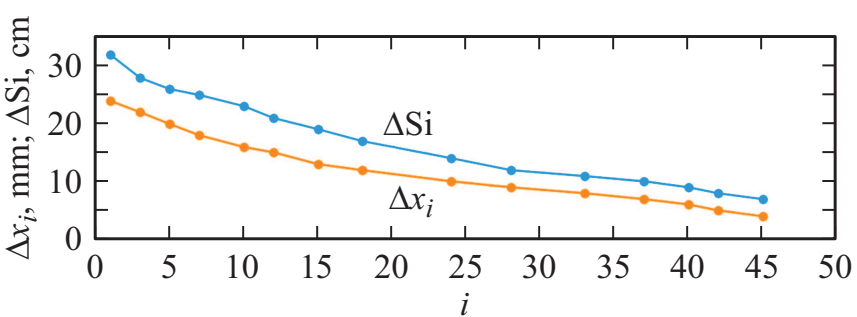

Рис. 11. Зависимость $\Delta x_{i}$ от номера пластины $i$. 


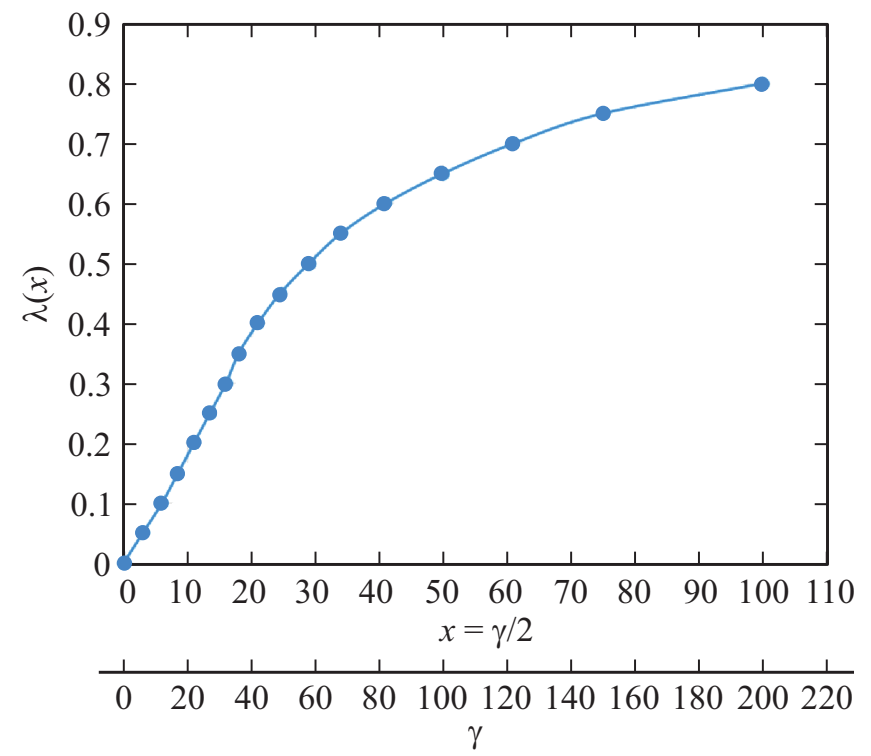

Рис. 12. Зависимость $\lambda$ от параметра $x$.

охлаждением; $\dot{J}_{i}=\frac{\Delta \dot{\gamma}_{i^{\prime}}\left[\lambda\left(\gamma_{i}\right)\right]}{\theta_{i}^{2}}-$ плотность потока энергии фотонов (отн. ед.); $\theta_{\gamma}=\theta_{e} C$, где $C-$ постоянная, зависящая от свойств материала мишени (для бесконечно тонкой мишени) [8]; $\theta_{e} \sim \frac{1}{\gamma} ; \theta_{\gamma} \approx \theta_{e}$. Электрон после излучения фотона движется под таким же средним углом относительно первоначального направления [9].

Пересчет от угла для „бесконечно тонкой мишени“ к мишени при кратных и при многократных столкновениях может быть сведен к простому введению коэффициента, зависящего от толщины мишени $t$ и вещества мишени [8]. Так что его введение ничего не меняет в формуле (16) - в числителе и знаменателе один и тот же коэффициент сокращается. Здесь, справедливости ради, необходимо отметить, что величина $t$ одинакова (или меняется незначительно при разных актах прохождения электронов, т. е. для орбит разного радиуса и энергии).

Площадь потока фотонов $S_{i}$ пропорциональна $D_{i}$ и $\frac{1}{\gamma_{i}^{2}}$. Значения $\lambda\left(\gamma_{i}\right)$ могут быть вычислены по аппроксимирующей формуле

$$
\lambda\left(\gamma_{i}\right) \approx 0.08487 \sqrt{\gamma_{i}}-2 \cdot 10^{-3} \gamma_{i},
$$

которая может быть выведена на основе нахождения коэффициентов приближенной зависимости вида $\lambda(x)=a \sqrt{x}+b x$ из графика $\lambda(x)$, построенного исходя из нескольких известных значений [9] - (рис. 12).

$$
\lambda\left(\gamma_{i}\right)=\frac{\left(\frac{d E}{d x}\right)_{\mathrm{rad}}}{\left(\frac{d E}{d x}\right)_{\mathrm{rad}}+\left(\frac{d E}{d x}\right)_{i o n}} .
$$

Значения $\left(\frac{d E}{d x}\right)_{r a d}$ и $\left(\frac{d E}{d x}\right)_{i o n}$ берутся для текущих значений энергии $\left(E=E_{0} \gamma_{i}\right.$, где $E_{0}=0.511 \mathrm{MeV}$ - энергия покоя электрона).

Потери на синхротронное излучение пренебрежимо малы, и составляют всего около $0.045 \mathrm{keV}$ на оборот при энергии $100 \mathrm{MeV}$ и радиусе поворота $0.2 \mathrm{~m}$ [10].
На рис. 13 показаны зависимости $Q_{\gamma_{i}}$ для случаев с охлаждением и без охлаждения (со звездочкой).

На рис. 14 показана зависимость $K_{I}$ от числа орбит, из которой видно, что увеличение $\dot{I}$ более 35 не дает существенного увеличения выхода фотонов в данном варианте, т.е. можно было бы рассчитывать, например, не на 46, а на 35 орбит, если учитывать только фактор, связанный с охлаждением.

Однако этот коэффициент $K_{\dot{I}}$ должен был бы быть умножен на коэффициент $K_{i}^{\prime}$, учитывающий эффект, связанный с отсутствием поглощения и рассеяния фотонов в веществе мишени, как в традиционном варианте с единственной мишенью оптимальной толщины.

Данный коэффициент можно оценить, сравнивая угол расхождения пучка излучения в двух случаях - без учета данного факта, т.е. рассеяния в веществе конверсионной мишени, и с учетом этого факта. Расчет по программам, апробированным сравнением с экспериментом [6], дает следующие усредненные значения угла:

1) толщина медной мишени $0.7 \mathrm{~mm}$ - от 1.6 до $36^{\circ}$, $14 \mathrm{~mm}-4^{\circ}$.

В нашем расчете без учета фактора рассеяния в меди:

1) $0.7 \mathrm{~mm}-0.4^{\circ}, 14 \mathrm{~mm}-4^{\circ}$.

Коэффициент усиления $\bar{K}_{\dot{I}}^{\prime}$ может достигать величины в диапазоне 16-50. При самом „скромном“ варианте $\left(\bar{K}_{\dot{I}}^{\prime}=16\right)$ можно рассчитывать на совокупный эффект для 10 фольг $\bar{K}_{\Sigma} \approx 10-12$ ( $K_{\dot{I}}^{\prime}$ выбран равным 5, так как 10 фольг примерно соответствуют четверти расстояния, проходимого фотонами в 46 фольгах).

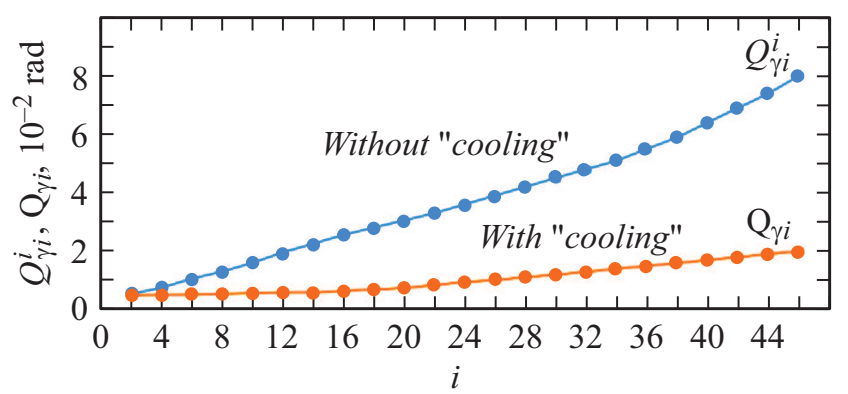

Рис. 13. Зависимость $Q_{\gamma_{i}}$ и $Q_{\gamma_{i}}^{*}$ от номера $i$.

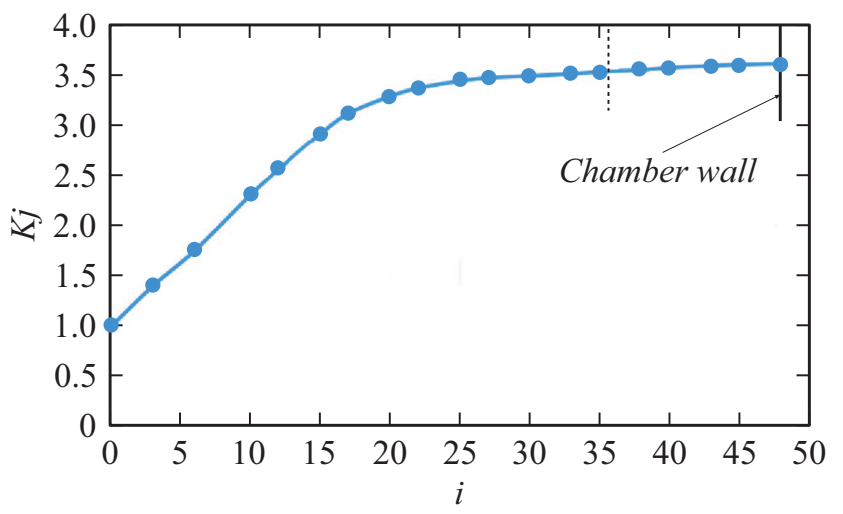

Рис. 14. Зависимость $K_{j}$ от $j$. 


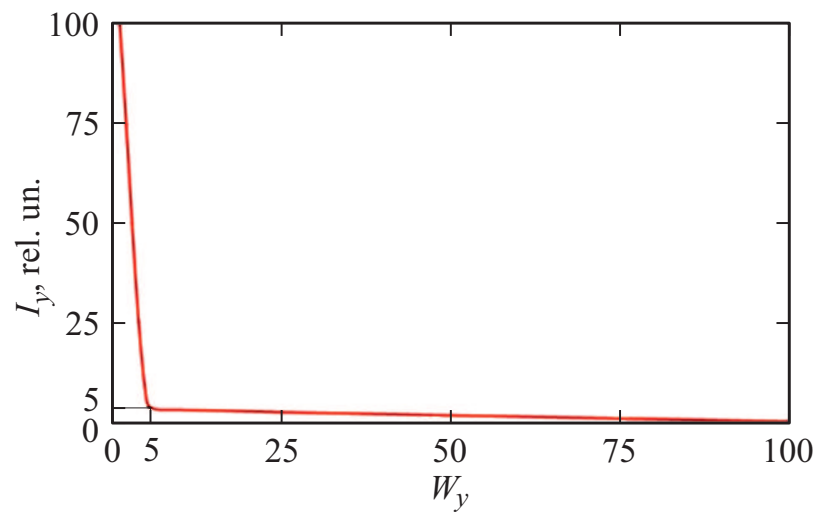

Рис. 15. Энергетический спектр тормозного излучения от электронов с энергией $W_{e}=100 \mathrm{MeV}, \Sigma \Delta m_{i}=15 \mathrm{~mm}$, $f\left(x^{\prime}\right) \approx 5 \cdot 10^{-2}, x^{\prime} \approx 5 \cdot 10^{-2}, \frac{\left\langle W_{\gamma}\right\rangle}{W_{\gamma \max }} \approx 1.14 \cdot 10^{-2}$.

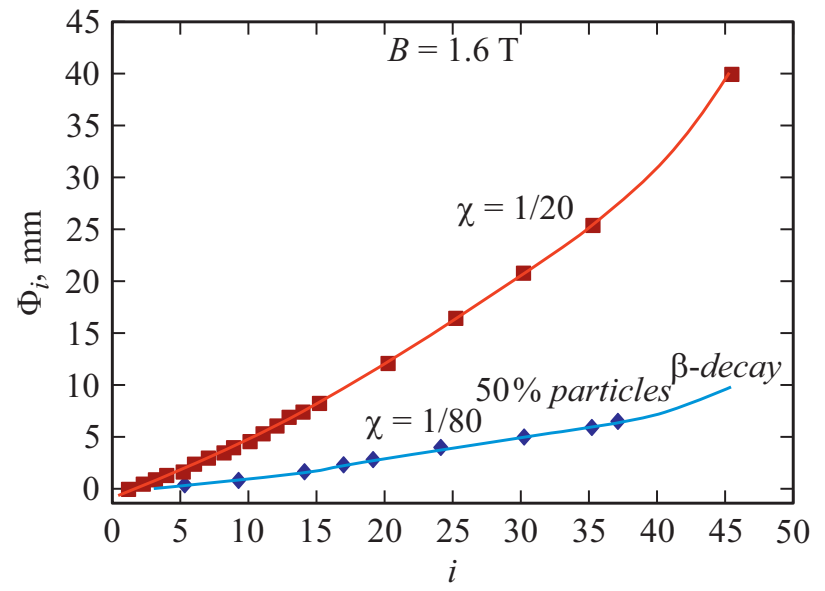

Рис. 16. Изменение диаметра пучка электронов с номером $i$ (при $i=35, \Delta m_{i}=393[\mu \mathrm{m}], E_{35}=35 \mathrm{MeV}, \Delta m_{35}=310[\mu \mathrm{m}]$, $\left.\frac{E_{1 \max }}{E_{\max }}=0.35\right)$.

Оценим, как меняются размеры электронного пучка в межполюсном зазоре магнита (вакуумной камере) по мере прохождения через фольги (рассеяние) и „раздувания" пучка при вращении. Наиболее критично это в направлении оси ,z“, так как пучок может высаживаться на стенки камеры, теряя интенсивность.

На рис. 15 показан один из характерных спектров фотонов тормозного излучения.

Анализ спектров для множества сочетаний параметров в рассматриваемом диапазоне показал, что $\chi$ изменяется приблизительно от $1 / 20$ до $1 / 80$. На рис. 16 показаны зависимости изменения диаметра пучка электронов $\Phi_{i}$ от числа оборотов при индукции $1.6 \mathrm{~T}$ (в 2 раза больше, чем ранее). Это сделано потому, что в первом варианте пучок начинает касаться стенки и теряется по мере вращения. При расчетах использованы выражения

$$
\Phi_{i}=\chi \sum_{i^{\prime}=1}^{i} S_{i^{\prime}} \sum_{i^{\prime \prime}=1}^{i^{\prime}}\left(\frac{1}{\gamma_{i^{\prime \prime}}}\right)^{2}
$$

$$
\begin{gathered}
\left(\Theta_{e}\right)_{i}=\chi \sqrt{\sum_{i^{\prime}=1}^{i}\left(\frac{1}{\gamma_{i^{\prime}}}\right)^{2}}, \\
\left(\Theta_{e}\right)_{1}=\sqrt{\left(\Theta_{e 1}^{0}\right)^{2}+\left(\Theta_{e 0}\right)^{2}}=\Theta_{e 1}^{0}=\left(\Theta_{\gamma}\right)_{1} \chi=\frac{1}{\gamma_{1}}, \\
\Phi_{i}=\Phi_{i-1}+S_{i}\left(\Theta_{e}\right)_{i^{\prime}} ; \quad\left(S_{i}=\pi D_{i}\right) .
\end{gathered}
$$

Это, однако, не влияет на результаты оценки $K_{i}$ для бесконечно тонкой мишени.

Рассчитаем толщины фольг $\Delta m_{i}$ для каждой из траекторий. Для этого используем соотношения

$$
\Delta m_{i}=\frac{\Delta E_{i}}{\rho\left(\frac{d E_{i}}{d x}\right)_{\Sigma}},
$$

где $\rho-$ плотность вещества мишени;

$$
\left(\frac{d E_{i}}{d x}\right)_{\Sigma}=\left(\frac{d E_{i}}{d x}\right)_{i o n}+\left(\frac{d E_{i}}{d x}\right)_{r a d}
$$

- суммарное значение потерь энергии электрона на ионизацию и излучение, значения которых табулированы [9].

На рис. 17 показана зависимость $\left(\frac{d E_{i}}{d x}\right)_{\Sigma}$ от $\gamma$, при расчетах использована аппроксимация

$$
\frac{d E_{i}}{d x}\left(\gamma_{i}\right) \approx 2.76+3.75 \cdot 10^{-2}\left(\gamma_{i}-40\right) \text {. }
$$

При этом использованы соотношения

$$
\begin{aligned}
& \frac{d E\left(\gamma_{i}\right)}{d x}=2.76+\xi\left(\gamma_{i}-40\right), \\
& \xi=\frac{(8.756-2.760)}{160}=3.75 \cdot 70^{-2}, \\
& \frac{b}{d}=\frac{a}{c} \rightarrow b=\frac{a d}{c}, \\
& c=200-40=160, \\
& a=6.756-2.76, \\
& b=\gamma_{i}-40 .
\end{aligned}
$$

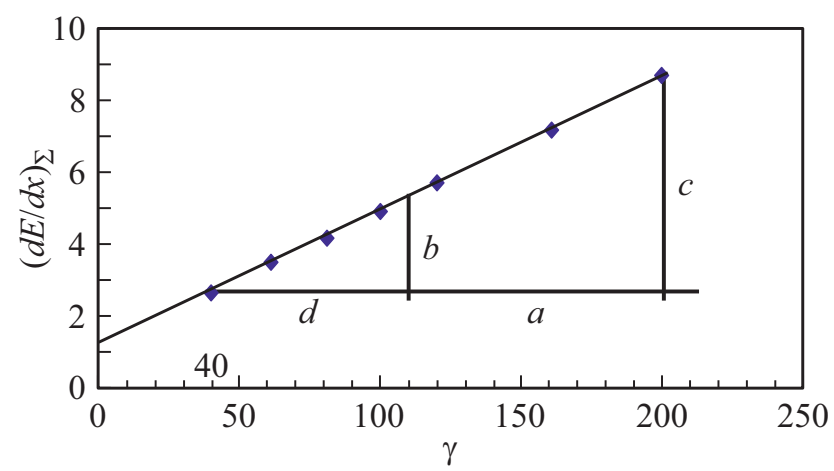

Рис. 17. Зависимость суммарных потерь энергии электрона от $\gamma$ (аппроксимация). 


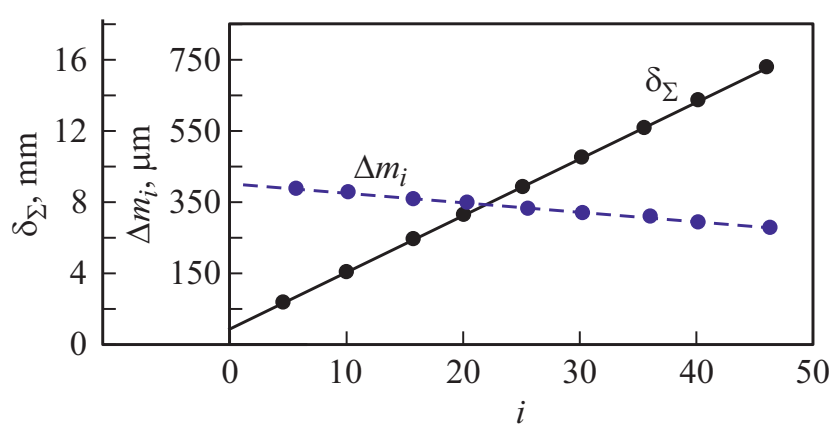

Рис. 18. Зависимость толщины меди при вращении пучка от номера орбиты электрона.

Значения толщин меди $\Delta m_{i}$, соответствующих каждому прохождению, приведены на рис. 18. Там же показано изменение полной толщины меди $\delta_{\Sigma}$, преодолеваемой электронами после прохождения ,i“ фольг (одной фольги разной толщины в разных местах).

Коэффициент конверсии энергии электронов в излучение составляет $\sim 75 \%$ для меди и $\sim 85 \%$ для вольфрама.

\section{Заключение}

Расчет динамики частиц в системе формирования пучка тормозного излучения на выходе ускорителя показал, что задача повышения интенсивности излучения может быть успешно решена, что особенно важно для ускорителей на энергию более $10 \mathrm{MeV}$ (до $100 \mathrm{MeV}$ и более). В частности, согласно результатам расчета, интенсивность может быть увеличена как минимум в несколько раз. Проведенные вычисления дают основания для проектирования систем, обеспечивающих „просвечивание“ инспектируемого объекта на большую глубину, а область детектируемых элементов расширена, в частности, при детектировании взрывчатых веществ, за счет применения пучков с более высокой энергией, где применение известных коллимирующих устройств малоэффективно (отношение „сигнал-шум“ в них практически мало зависит от энергии).

\section{Конфликт интересов}

Авторы заявляют, что у них нет конфликта интересов.

\section{Список литературы}

[1] B.Yu. Bogdanovich, V.V. Kudinov, A.V. Nesterovich, E.A. Tsygankov, V.V. Janenko. Bulletin American Phys. Society, 42 (3), 1388 (1997).

[2] А.С. Белоусов, А.И. Карев, Е.И. Малиновский, В.Г. Граевский, Е.И. Тамм, Ю.А. Коняев, Ю.М. Кочегаров, В.П. Милованов, А.С. Румянцев, Р.Р. Илющенко, Р.Г. Майструк, Н.И. Ханюченко, А.А. Орлов, С.В. Твердохлебов. Наука производству, 6 (31), 33 (2000).
[3] S. Kutsaev, R. Agustsson, A. Arodzero, S. Boucher, J. Hartzell, A. Murokh, F. O'Shea, A.Yu. Smirnov. Phys. Proced., 90, 115 (2017). DOI: https://doi.org/10.1016/j.phpro.2017.09.036

[4] Б.Ю. Богданович, А.В. Нестерович, А.Е. Шиканов, М.Ф. Ворогушин, Ю.А. Свистунов. Дистанционный радиационный контроль с линейными ускорителями: в $2 \mathrm{~m}$. Линейные ускорители для генерации тормозного излучения и нейтронов (Энергоатомиздат, М., 2009), т. 1, $272 \mathrm{c}$.

[5] Б.Ю. Богданович, А.В. Нестерович, А.Е. Шиканов, М.Ф. Ворогушин, Ю.А. Свистунов. Дистанционный радиационный контроль с линейными ускорителями: в $2 \mathrm{~m}$. Комплексы радиационного контроля (Машиностроение, M., 2012), т. 2, 284 c.

[6] Б.Ю. Богданович, В.И. Каминский, В.В. Кудинов, А.В. Нестерович, В.А. Сенюков. Атомная энергия, 115 (1), 37 (2013).

[7] А.Н. Лебедев, А.В. Шальнов. Основы физики и техники ускорителей (Энергоиздат, М., 1981), т. 1, 192 с.

[8] Г. Штауб, Г.-А. Бете, Ю. Ашкин. Экспериментальная ядерная физика, под ред. Э. Сегре. (ИИЛ, М., 1955), т. 1, 662 с. [Пер. с англ.: G. Staub, G.-A. Bethe, Yu. Ashkin. Experimental Nuclear Physics, ed. by E. Segre. (John Wiley \& Sons, NY., 1953), v. 1, 789 p.]

[9] В.П. Ковалев. Вторичные излучения ускорителей электронов (Атомиздат, М., 1979, 198 с.)

[10] А.Н. Лебедев, А.В. Шальнов.Основы физики и техники ускорителей (Энергоиздат, М., 1982.), т. 2, 240 с. 